\title{
ESTUDO DA ESTABILIDADE DE MELÕES DESIDRATADOS OBTIDOS POR DESIDRATAÇÃO OSMÓTICA SEGUIDA DE SECAGEM CONVENCIONAL ${ }^{1}$
}

\author{
ANDRÉA DA SILVA LIMA², RAIMUNDO WILANE DE FIGUEIREDO ${ }^{3}$, GERALDO ARRAES MAIA ${ }^{4}$, JANICE RIBEIRO \\ LIMA $^{5}$, PAULO HENRIQUE MACHADO DE SOUSA ${ }^{6}$
}

\begin{abstract}
RESUMO - O presente trabalho teve por objetivo avaliar a estabilidade de melões desidratados obtidos por desidratação osmótica à pressão atmosférica $(760 \mathrm{mmHg})$ e a vácuo parcial $(660 \mathrm{mmHg})$ seguida de secagem convencional. A estabilidade dos produtos foi avaliada segundo suas características físico-químicas, microbiológicas e sensoriais, durante 180 dias de armazenamento, à temperatura ambiente. Ambos os processos resultaram em boa estabilidade físico-química e microbiológica dos produtos que mostraram boa aceitação durante todo o período de armazenamento. Termos para indexação: Cucumis melo L., processamento, tratamento osmótico, estabilidade.

\section{STABILITY EVALUATION IN DEHYDRATED MELONS OBTAINED BY A COMBINATION OF OSMOTIC DEHYDRATION AND CONVENTIONAL DRYING}

\begin{abstract}
The objective of the present work was to evaluate storage stability of melons processed by a previous osmotic dehydration at atmospheric pressure $(760 \mathrm{mmHg})$ or partial vacuum $(660 \mathrm{mmHg})$ followed by conventional drying. The stability was assessed monthly by means of physico-chemical, microbiological and sensorial methods, during 180 days of storage at room temperature. Both the processes resulted in good physico-chemical and microbiological stability of the products, which presented good acceptability during all the storage time.

Index terms: Cucumis melo L., processing, osmotic treatment, stability.
\end{abstract}

\section{INTRODUÇÃO}

Na atualidade, a demanda por produtos naturais, saudáveis e à base de frutas tem crescido rapidamente, não apenas como produtos acabados, mas também como ingredientes a serem incluídos em alimentos mais elaborados, como sorvetes, cereais, laticínios, produtos de confeitaria e panificação. O tratamento osmótico tem se apresentado como uma ferramenta tecnológica importante para se desenvolverem novos produtos derivados de frutas, com valor agregado e com propriedades funcionais (Torreggiani \& Bertolo, 2001).

Tratamentos osmóticos estão sendo usados principalmente como um pré-tratamento introduzido em alguns processos convencionais, tais como secagem a ar convectivo, microondas e liofilização, a fim de melhorar a qualidade do produto final, reduzir custos de energia ou mesmo formular novos produtos (Sereno et al., 2001). Segundo Maestrelli et al. (2001), o uso da desidratação osmótica, combinada ou não a outros processos, tem sido efetivo na redução do colapso estrutural de frutos delicados, mesmo quando posteriormente se aplicam processos agressivos, como tratamento térmico. Além disso, o tratamento osmótico, em certas condições, pode favorecer a retenção dos pigmentos da fruta, evitar o escurecimento enzimático e fornecer produtos mais atraentes em termos de aparência para o consumo (Krokida et al., 2000).

A desidratação osmótica permite tanto a remoção de água do produto quanto a modificação de suas propriedades pela impregnação de solutos desejados (Mizrahi et al., 2001). Essa técnica emprega soluções de alta pressão osmótica, em que dois fluxos são estabelecidos: a saída de água do alimento para a solução e a incorporação do soluto pelo alimento, devido aos gradientes de concentração. Nesse sentido, o prétratamento osmótico pode melhorar aspectos nutricionais, sensoriais e funcionais dos alimentos, sem comprometer sua integridade, sendo efetivo mesmo à temperatura ambiente, de maneira que o dano térmico à textura, cor e aroma do alimento é minimizado (Torreggiani, 1993).

A utilização de vácuo durante a desidratação osmótica apresenta-se como um avanço dentro do processo, contribuindo para acelerar a perda de água, em comparação com o tratamento a pressão atmosférica, tornando o processo mais rápido e possibilitando a obtenção de frutos desidratados de boa qualidade (Shi et al., 1995).
O presente trabalho teve por objetivo avaliar a estabilidade de melões desidratados obtidos por desidratação osmótica sob pressão atmosférica e sob vácuo seguida de secagem convencional. A estabilidade dos produtos foi avaliada segundo suas características físico-químicas, microbiológicas e sensoriais, a cada trinta dias, durante 180 dias de armazenamento do produto à temperatura ambiente.

\section{MATERIALEMÉTODOS}

Os melões (Cucumis melo L. c.v. Cantaloupe) utilizados para o desenvolvimento do trabalho foram adquiridos em mercado varejista de Fortaleza-CE, vindos da CEASA, em estádio comercial de maturação.

Os xaropes osmóticos foram preparados utilizando açúcar cristalizado granulado, adquirido em mercado local. Os conservantes químicos adicionados ao xarope foram: ácido cítrico de grau alimentício (monoidratado), fabricado pela FERMENTA LTDA-SP, para corrigir o pH, e benzoato de sódio de grau alimentício (98\%), fabricado por LIQUID QUÍMICA S.A. - Liquid Carbonic, para a estabilização do produto final. Os xaropes de sacarose foram preparados por meio da adição de açúcar à água (até atingir o teor de sólidos solúveis desejado), sob aquecimento, para facilitar a dissolução. Em seguida, foram adicionados ácido cítrico (quantidade necessária para se atingir $\mathrm{pH} 3,0$ ) e benzoato de sódio (concentração de $0,1 \%$ ).

Os frutos foram selecionados, lavados em água clorada (50ppm de cloro ativo/15min), descascados e cortados manualmente em cubos (aproximadamente 3,0 cm). Em seguida, foram branqueados em vapor saturado fluente $\left(100^{\circ} \mathrm{C} / 2 \mathrm{~min}\right)$ e submetidos a um dos tratamentos osmóticos descritos a seguir:

Tratamento 1: xarope de sacarose a $65^{\circ}$ Brix, na proporção fruto:xarope de 1:2 e pressão atmosférica $(760 \mathrm{mmHg})$, por 5 horas.

Tratamento 2: xarope de sacarose a $65^{\circ} \mathrm{Brix}$, na proporção fruto:xarope de 1:4 e vácuo $(660 \mathrm{mmHg})$, por 3 horas.

Os frutos foram, então, imersos em soluções de sacarose a $65^{\circ}$ Brix e mantidos sob osmose à temperatura de $65^{\circ} \mathrm{C}$ em um tacho em aço inoxidável, com camisa de aquecimento por resistência elétrica, e sistema de vácuo por bomba d'água.

Ao final do processo osmótico, de ambos os tratamentos, os

\footnotetext{
${ }^{1}$ (Trabalho 110/2003). Recebido: 04/09/2003; Aceito para publicação: 05/03/2004. Parte da Dissertação de Mestrado do primeiro autor apresentada ao DTA/UFC. Apoio BNB.

${ }^{2}$ Quím. Industrial; M.Sc., Dep. Tecnologia de Alimentos, DTA/UFC, CP 12168, CEP: 60356-000, Fortaleza-CE. E-mail: andreadasilvalima@ yahoo.com.br ${ }^{3}$ Eng. Agr., D.Sc., Dep. Tecnologia de Alimentos, DTA/UFC, E-mail: figueira@ ufc.br

${ }^{4}$ Eng. Agr., Ph.D., Dep. Tecnologia de Alimentos, DTA/UFC, E-mail: frutos@ ufc.br

${ }_{5}^{5}$ Pesquisadora da Embrapa Agroindústria Tropical - R. Dra Sara Mesquita, 2270 - Planalto Pici - CEP: 60511-110, Fortaleza - Ceará. janice@ cnpat.embrapa.br

${ }^{6}$ Quím., M.Sc., Dep. Tecnologia de Alimentos, DTA/UFC, E-mail: phmachado@uol.com.br
} 
frutos foram dispostos em bandejas e colocados em estufa com circulação forçada de ar, à temperatura de $65^{\circ} \mathrm{C}$, durante 12 horas. Ao término do processo, os produtos foram acondicionados em embalagens flexíveis de polipropileno biorentado (BOPP), cada uma contendo $120 \mathrm{~g}$ do produto, $\mathrm{e}$ armazenados à temperatura ambiente, por um período de 180 dias.

A estabilidade dos produtos elaborados foi avaliada por meio de análises físico-químicas (atividade de água, umidade, $\mathrm{pH}$, acidez total titúlavel, sólidos solúveis totais, açúcares redutores e não redutores, cor e textura), microbiológicas (contagens de microrganismos aeróbios mesófilos, bolores e leveduras, coliformes a $35^{\circ} \mathrm{C}$ e a $45^{\circ} \mathrm{C}$, e Salmonella sp.) e sensorial (teste de aceitação global). Os produtos foram analisados a cada 30 dias, por um período de 180 dias.

A determinação da atividade de água das amostras foi realizada em aparelho digital AQUALAB - Decagon Devices Inc. EUA, modelo CX-2 (sensibilidade 0,001), à temperatura ambiente. A umidade foi determinada por evaporação direta em estufa MARCONI, modelo MA 035, à temperatura de $70^{\circ} \mathrm{C}$ (Instituto Adolfo Lutz, 1985). $\mathrm{O} \mathrm{pH}$ foi determinado por meio de um potenciômetro HANNA INSTRUMENTS, modelo HI 9321 (calibrado periodicamente com soluções tampão de $\mathrm{pH}$ 4,0 e 7,0). A acidez total titulável foi determinada em um titulador potenciométrico METTLER, modelo DL12 (calibrado periodicamente com soluções tampão de pH 4,0 e 7,0). O teor de sólidos solúveis totais das amostras foi determinado por refratometria (Instituto Adolfo Lutz, 1985). Os açúcares redutores foram determinados pelo método do ácido dinitrosalicílico (Miller, 1959) e os não redutores conforme Instituto Adolfo Lutz (1985). A cor foi determinada por meio de leituras diretas realizadas em colorímetro MINOLTA, modelo CR300, com valores expressos em $\mathrm{L}^{*}$. As análises microbiológicas foram realizadas conforme a metodologia descrita por Downes \& Ito (2001). A avaliação sensorial foi feita por meio do teste de aceitação global, utilizando-se de uma escala hedônica estruturada de 9 pontos, onde 1 representava a nota mínima (desgostei muitíssimo) e 9 a nota máxima (gostei muitíssimo), aplicada a 32 provadores não treinados (Monteiro, 1984).

Os resultados das análises físico-químicas e sensoriais foram avaliados estatisticamente quanto à análise de variância, e a comparação de médias, pelo teste de Tukey (SAS, 1996).

\section{RESULTADOS E DISCUSSÃO}

Os valores de atividade de água, umidade, $\mathrm{pH}$, acidez total titulável e de sólidos solúveis totais, obtidos no decorrer do armazenamento, não apresentaram diferença significativa $(p>0,05)$ com o tempo, para ambos os produtos (Tabela 1). Entretanto, a análise estatística dos valores de açúcares obtidos em função do tempo de armazenamento mostrou existir correlação significativa $(\mathrm{p}<0,001)$ com o tempo, para ambos os produtos, tendo sido observado um decréscimo no teor de açúcares não redutores e um acréscimo no teor de açúcares redutores a partir do terceiro mês de estocagem dos produtos (Figuras 1 e 2). A incorporação de sacarose durante a desidratação osmótica do fruto levou a uma predominância deste açúcar não redutor no produto final. Durante o armazenamento, a acidez do meio possivelmente propiciou a hidrólise da sacarose, levando à formação de glicose e frutose.
Observações semelhantes foram reportadas por Souza Neto (2002) na desidratação osmótica de manga à pressão atmosférica e a vácuo.

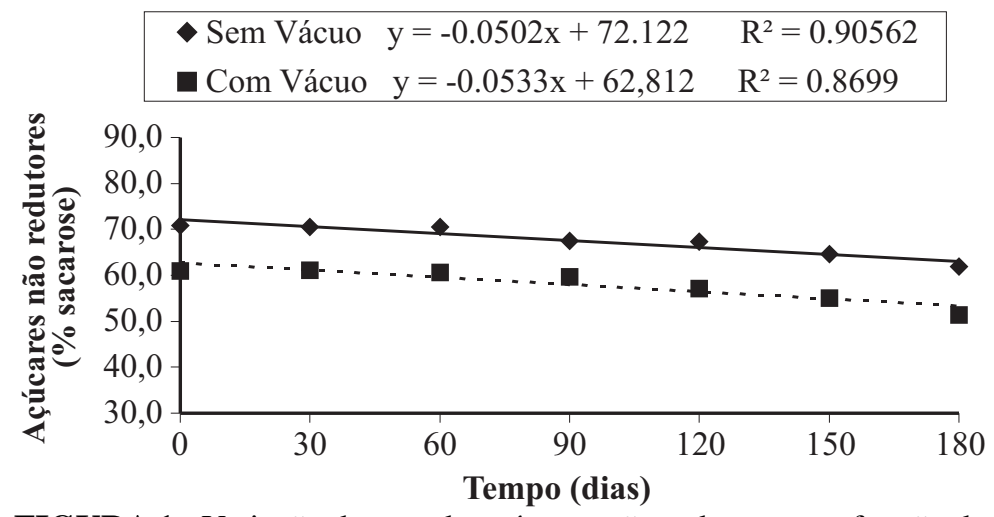

FIGURA 1 - Variação do teor de açúcares não redutores em função do tempo de armazenamento dos produtos.

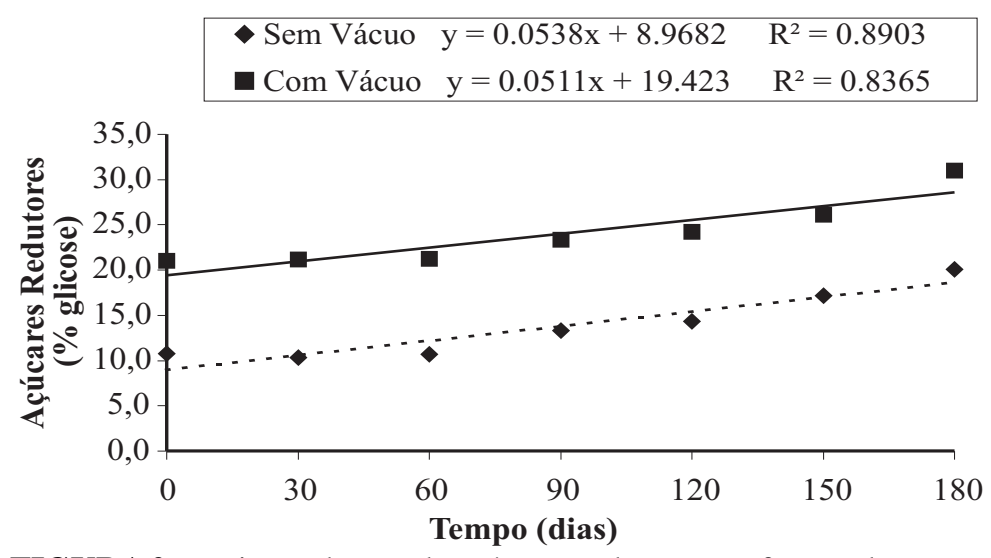

FIGURA 2 - Variação do teor de açúcares redutores em função do tempo de armazenamento dos produtos.

Os valores obtidos para cor, no decorrer do armazenamento, apresentaram diferença significativa, a $\mathrm{p}<0,05$ para o produto pré-tratado osmoticamente a pressão atmosférica, e $\mathrm{p}<0,001$ para o produto pré-tratado osmoticamente a vácuo, indicando maior tendência ao escurecimento (redução do valor de L*) com o tempo de armazenamento (Figura 3). Essa mudança de cor pode estar relacionada ao escurecimento não enzimático, que ocorre intensamente na presença de açúcares e nas faixas de umidade de $14 \%$ - $17 \%$ e atividade de água de 0,65 - 0,74 nos produtos obtidos. Sousa (2002) também observou, em estudos com bananas desidratadas por pré-tratamento osmótico à pressão atmosférica e a vácuo, seguido de secagem em estufa, diminuição no valor de L* com o decorrer do tempo de estocagem dos produtos à temperatura ambiente.

Os produtos obtidos, nos dois tratamentos, logo após o processamento e em todos os tempos de armazenamento avaliados, apresentaram contagens de bolores e leveduras inferiores a $100 \mathrm{UFC} / \mathrm{g}$ e coliformes a $35^{\circ} \mathrm{C}$ e a $45^{\circ} \mathrm{C}$ inferiores a $3 \mathrm{NMP} / \mathrm{g}$. A presença de Salmonella

TABELA 1 - Análises físico-químicas de amostras de melão obtido por desidratação osmótica a pressão atmosférica $\left(\mathrm{P}_{\mathrm{A}}\right)$ e a vácuo (V), em solução de sacarose a $65^{\circ}$ Brix e proporções fruto:xarope 1:2 e 1:4, respectivamente, seguidas de secagem em estufa.

\begin{tabular}{|c|c|c|c|c|c|c|c|c|c|c|}
\hline \multirow{2}{*}{$\begin{array}{l}\text { Tempo } \\
\text { (dias) }\end{array}$} & \multicolumn{2}{|c|}{ Aa } & \multicolumn{2}{|c|}{ U (\%) } & \multicolumn{2}{|c|}{ PH } & \multicolumn{2}{|c|}{$\begin{array}{c}\mathrm{ATT} \\
\text { (g ác. cítrico/100g) }\end{array}$} & \multicolumn{2}{|c|}{$\begin{array}{c}\text { SST } \\
\left({ }^{\circ} \mathrm{BRIX}\right)\end{array}$} \\
\hline & $\mathbf{P}_{\mathbf{A}}$ & V & $\mathbf{P}_{\mathbf{A}}$ & $\mathbf{V}$ & $\mathbf{P}_{\mathbf{A}}$ & V & $\mathbf{P}_{\mathrm{A}}$ & V & $\mathbf{P}_{\mathbf{A}}$ & V \\
\hline 0 & $0,733^{\mathrm{a}}$ & $0,651^{\mathrm{a}}$ & $16,24^{\mathrm{a}}$ & $14,10^{\mathrm{a}}$ & $5,39^{\mathrm{a}}$ & $5,64^{\mathrm{a}}$ & $0,317^{\mathrm{a}}$ & $0,377^{\mathrm{a}}$ & $82,60^{a}$ & $83,40^{\mathrm{a}}$ \\
\hline 30 & $0,734^{\mathrm{a}}$ & $0,660^{\mathrm{a}}$ & $16,53^{\mathrm{a}}$ & $15,13^{\mathrm{a}}$ & $5,45^{\mathrm{a}}$ & $5,57^{\mathrm{a}}$ & $0,310^{\mathrm{a}}$ & $0,384^{\mathrm{a}}$ & $83,67^{\mathrm{a}}$ & $84,40^{\mathrm{a}}$ \\
\hline 60 & $0,733^{\mathrm{a}}$ & $0,654^{\mathrm{a}}$ & $15,67^{\mathrm{a}}$ & $14,52^{\mathrm{a}}$ & $5,42^{\mathrm{a}}$ & $5,60^{\mathrm{a}}$ & $0,338^{\mathrm{a}}$ & $0,383^{\mathrm{a}}$ & $83,73^{\mathrm{a}}$ & $84,80^{\mathrm{a}}$ \\
\hline 90 & $0,735^{\mathrm{a}}$ & $0,657^{\mathrm{a}}$ & $16,28^{\mathrm{a}}$ & $14,83^{\mathrm{a}}$ & $5,46^{\mathrm{a}}$ & $5,62^{\mathrm{a}}$ & $0,343^{\mathrm{a}}$ & $0,401^{\mathrm{a}}$ & $82,33^{\mathrm{a}}$ & $84,87^{\mathrm{a}}$ \\
\hline 120 & $0,734^{\mathrm{a}}$ & $0,667^{\mathrm{a}}$ & $16,52^{\mathrm{a}}$ & $14,36^{\mathrm{a}}$ & $5,40^{\mathrm{a}}$ & $5,65^{\mathrm{a}}$ & $0,324^{a}$ & $0,384^{\mathrm{a}}$ & $83,13^{\mathrm{a}}$ & $83,20^{\mathrm{a}}$ \\
\hline 150 & $0,734^{\mathrm{a}}$ & $0,651^{\mathrm{a}}$ & $16,49^{\mathrm{a}}$ & $14,06^{\mathrm{a}}$ & $5,37^{\mathrm{a}}$ & $5,55^{\mathrm{a}}$ & $0,330^{\mathrm{a}}$ & $0,389^{\mathrm{a}}$ & $82,47^{\mathrm{a}}$ & $85,00^{\mathrm{a}}$ \\
\hline 180 & $0,732^{\mathrm{a}}$ & $0,664^{\mathrm{a}}$ & $16,08^{\mathrm{a}}$ & $14,67^{\mathrm{a}}$ & $5,35^{\mathrm{a}}$ & $5,58^{\mathrm{a}}$ & $0,311^{\mathrm{a}}$ & $0,393^{\mathrm{a}}$ & $83,67^{\mathrm{a}}$ & $83,80^{\mathrm{a}}$ \\
\hline
\end{tabular}

Os valores das médias seguidos das mesmas letras, na mesma coluna, não diferem ao nível de 5\%, pelo teste de Tukey; Aa: atividade de água; U: umidade; ATT: acidez total titulável; SST: sólidos solúveis totais; ANR: açúcares não redutores; AR: açúcares redutores. 


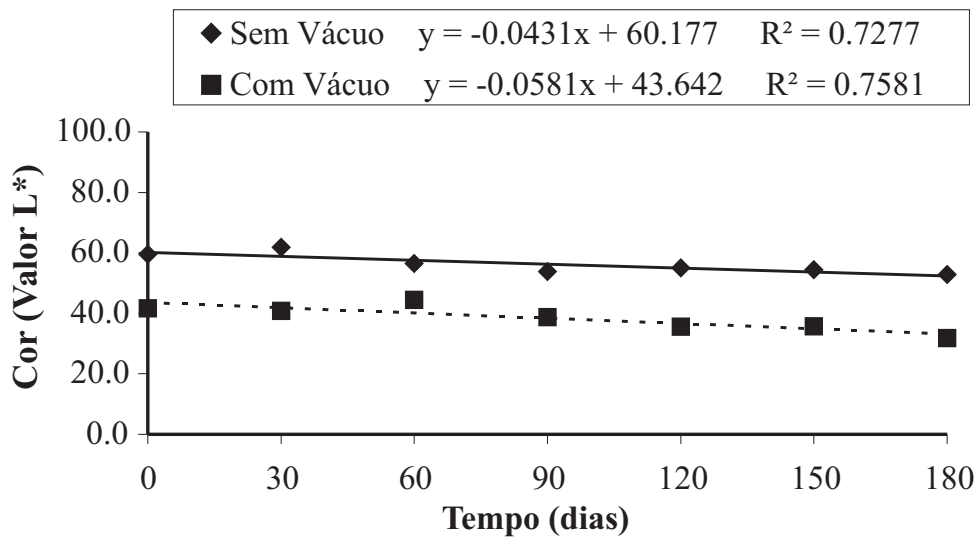

FIGURA 3 - Variação da cor em função do tempo de armazenamento dos produtos.

sp. não foi detectada em nenhuma das amostras analisadas. Entretanto, foram encontrados valores de bactérias aeróbias mesófilas que variaram entre $2,43 \times 10^{2}$ e $7,13 \times 10^{2} \mathrm{UFC} / \mathrm{g}$ para o produto pré-tratado osmoticamente à pressão atmosférica e entre $1,2 \times 10^{3} \mathrm{e} 1,8 \times 10^{4} \mathrm{UFC} / \mathrm{g}$ para o produto pré-tratado a vácuo. Desta forma, os produtos atenderam aos padrões microbiológicos estabelecidos pela legislação federal vigente (Anvisa, 2001). Não foi observado o crescimento de coliformes a $45^{\circ} \mathrm{C}$, sendo estes indicadores da possível presença de enteropatógenos, como a Salmonella sp. Estes dados sugerem as boas condições higiênicosanitárias dos produtos elaborados.

A contagem de bolores e leveduras, bactérias aeróbias mesófilas, e a pesquisa de coliformes a $35^{\circ} \mathrm{C}$ e a $45^{\circ} \mathrm{C}$ apresentaram valores que não comprometeram a estabilidade dos produtos. Estes resultados obtidos após o processamento e durante o armazenamento podem ser atribuídos às características dos produtos, que possuem baixos níveis de atividade de água aliados à presença de aditivos [benzoato de sódio $(0,1 \%)$ e ácido cítrico ( $\mathrm{pH} 3,0)$ ], que os tornaram desfavoráveis ao crescimento microbiano, principalmente mofos e leveduras, que são considerados os microrganismos responsáveis pela deterioração de alimentos de umidade intermediária (Hocking, 1988).

Os valores das médias das notas obtidas nos testes de aceitação global foram de 6,8 e 5,8 (para o começo e final da armazenagem) para o produto pré-tratado osmoticamente à pressão atmosférica e de 7,3 e 6,2 para o produto pré-tratado a vácuo. As médias das notas de aceitação obtidas no decorrer do armazenamento não apresentaram diferença significativa $(p>0,05)$ com o tempo, para ambos os produtos. A análise comparativa das médias de aceitação global dos dois produtos elaborados, em cada tempo de armazenamento, mostrou também não haver diferença significativa $(p>0,05)$ entre os tratamentos. Observouse ainda que as médias para este atributo mantiveram-se, durante todo o período de armazenamento, próximos de 6,0, o que corresponde, na escala hedônica, a "gostei ligeiramente". Segundo Labuza \& Schimdl (1988), o final da vida útil de um produto pode ser considerado quando há uma queda de 1,5 ponto na escala hedônica, o que não ocorreu com os produtos durante o armazenamento. Isto indica que os produtos podem ser considerados como aceitáveis pelos consumidores por todo o tempo de vida de prateleira estudado.

\section{CONCLUSÕES}

Os produtos obtidos por desidratação osmótica sob pressão atmosférica e sob vácuo, seguida de secagem convencional, mantiveram suas características físico-químicas com pouca variação durante o armazenamento. Estes apresentaram boa estabilidade microbiológica durante os 180 dias de armazenamento, confirmando, assim, a eficiência da metodologia empregada na elaboração dos produtos de melões desidratados. A avaliação sensorial mostrou que os produtos tiveram boa aceitabilidade durante todo o período de armazenamento, e não apresentaram diferenças significativas entre os tratamentos.

\section{AGRADECIMENTOS}

Agradecimentos ao Departamento de Tecnologia de Alimentos da Universidade Federal do Ceará, à Funcap, à Embrapa Agroindústria Tropical e ao Banco do Nordeste, pelo suporte financeiro.

\section{REFERÊNCIASBIBLIOGRÁFICAS}

ANVISA. Agência Nacional de Vigilância Sanitária. Resolução RDC n. ${ }^{\circ} 12$, de 02 de janeiro de 2001: Regulamento técnico sobre os padrões microbiológicos para alimentos. Diário Oficial da União, Brasília, 02-01-2001, P1-54. Disponível em: <http:// www.anvisa.gov.br>. Acesso em: 30 de dez. 2002.

DOWNES, F. P.; ITO, K. Compendium of methods for the microbiological examination of foods. 4.ed. Washington, DC: American Public Health Association, 2001.676p.

HOCKING, A. D. Moulds and yeasts associated with foods of reduced water activity: ecological interaction. In: SEOW, C.C. (Ed.) Food preservation by moisture control. New York: Elsevier, 1988. p. 5772.

INSTITUTO ADOLFO LUTZ. Normas analíticas do Instituto Adolfo Lutz. Métodos químicos e físicos para análises de alimentos. 3.ed. São Paulo, 1985. v. 1, 533p.

KROKIDA, M. K.; KARATHANOS, V.T.; MAROULIS,Z.B.; MARINOSKOURIS, D. Effect of osmotic dehydration on color and sorption characterístics of apple and banana. Drying Technology, New York, v. 18, n. 6, p. 937-950, 2000.

LABUZA, T. P.; SCHIMDL, M. K. Use of sensory data in the shelf life testing of foods: principles and graphical methods for evaluation. Cereals Foods World, St. Paul, v. 33, n. 2, p. 193-206, 1988.

MAESTRELLI,A.; SCALZO, R.; LUPI,D.;BERTOLO,G;TORREGGIANI, D. Partial removal of water before freezing: cultivar and pre-treatments as quality factors of frozen muskmelon (Cucumis melo, cv. reticulatus Naud). Journal of Food Engineering, Oxford, v. 49, n. 2, p. 255-260, 2001.

MILLER,G. L. Use of dinitrosalicycle acid reagent for determination of reducing sugars. Analytical Chemistry, Washington, n. 31, p. 226$248,1959$.

MIZRAHI, S.; EICHLER, S.; RAMON, O. Osmotic dehydration phenomena in gel systems. Journal of Food Engineering, Oxford, v. 49, n. 1, p. 87-96, 2001.

MONTEIRO, C. B. L. Técnicas de avaliação sensorial. 2. ed. Curitiba: UFPR/CEPPA, 1984. 101p.

SAS Institute. SAS user's Guide: statistical analysis system, Cary, N.C., 1996.

SERENO, A. M.; HUBINGER, M. D.; COMESAÑA, J. F.; CORREA, A. Prediction of water activity of osmotic solutions. Journal of Food Engineering, Oxford, v. 49, n. 1, p.103-114, 2001.

SHI, X. Q.; FITO, P.; CHIRALT, A. Influence of vacuum treatment on mass transfer during osmotic dehydration of fruits. Food Research International, Monticello, v. 28, n. 5, p. 445-454, 1995.

SOUSA, P. H. M. Desidratação osmótica de banana com e sem vácuo com complemento de secagem em estufa de circulação de ar. 2002. 90f. Dissertação (Mestrado em Tecnologia de Alimentos) Faculdade de Engenharia de Alimentos, Universidade Federal do Ceará, Fortaleza, 2002.

SOUZA NETO, M. A. Desidratação osmótica de manga Coité com e sem a utilização de vácuo com complemento de secagem em estufa. 2002. 65f. Dissertação (Mestrado em Tecnologia de Alimentos) Faculdade de Engenharia de Alimentos, Universidade Federal do Ceará, Fortaleza, 2002.

TORREGGIANI, D. Osmotic dehydration in fruit and vegetable processing. Food Research International, Monticello, v. 26, n. 1, p. 59-68, 1993.

TORREGGIANI, D.; BERTOLO, G. Osmotic pre-treatments in fruit processing: chemical, physical and structural effects. Journal of Food Engineering, Oxford, v. 49, n. 2, p. 247-253, 2001. 